See discussions, stats, and author profiles for this publication at: https://www.researchgate.net/publication/325577909

\title{
Can the REBT theory explain loneliness? Theoretical and clinical applications
}

Article in Cognitive Behaviour Therapy · June 2018

DOl: 10.1080/16506073.2018.1475505

\section{CITATIONS}

0

6 authors, including:

Philip Hyland

3 National University of Ireland, Maynooth

193 PUBLICATIONS 2,468 CITATIONS

SEE PROFILE

Thanos Karatzias

Edinburgh Napier University

162 PUBLICATIONS 2,474 CITATIONS

SEE PROFILE

\section{READS}

851

Grainne McGinty

National University of Ireland, Maynooth

2 PUBLICATIONS 12 CITATIONS

SEE PROFILE

Jamie Murphy

Ulster University

116 PUBlications 1,376 CITATIONS

SEE PROFILE

Some of the authors of this publication are also working on these related projects:

ACCESS TO INFANT AND MATERNAL HEALTH - AIM HEALTH View project

http://kps.org.pl/en View project 


\section{Can the REBT theory explain loneliness? Theoretical and clinical applications}

Philip Hyland, Gráinne McGinty, Thanos Karatzias, Jamie Murphy, Frédérique Vallières \& Joanna McHugh Power

To cite this article: Philip Hyland, Gráinne McGinty, Thanos Karatzias, Jamie Murphy, Frédérique Vallières \& Joanna McHugh Power (2018): Can the REBT theory explain loneliness? Theoretical and clinical applications, Cognitive Behaviour Therapy, DOI: 10.1080/16506073.2018.1475505

To link to this article: https://doi.org/10.1080/16506073.2018.1475505

\section{Published online: 05 Jun 2018.}

Submit your article to this journal $₫$

Џ Article views: 9

Q View related articles $\square$

View Crossmark data ¿ 


\title{
Can the REBT theory explain loneliness? Theoretical and clinical applications
}

\author{
Philip Hyland ${ }^{a, b}$ (D), Gráinne McGintya ${ }^{a}$ Thanos Karatziasc,d, Jamie Murphye (iD, \\ Frédérique Vallières ${ }^{b}$ (D) and Joanna McHugh Power ${ }^{a}$
}

aschool of Business, National College of Ireland, Dublin, Ireland; ${ }^{b}$ Centre for Global Health, School of Psychology, Trinity College Dublin, Ireland; 'NHS Lothian, Rivers Centre for Traumatic Stress, Edinburgh, UK;

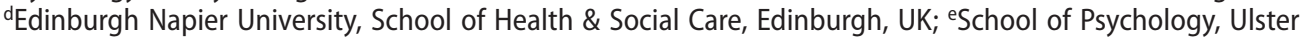
University, Derry, Northern Ireland

\begin{abstract}
Loneliness is a common psychological experience affecting a significant minority of the general population. Loneliness may in part be related to the existence of dysfunctional cognitive evaluations. To date, however, loneliness has yet to be explicitly assessed within a cognitive-behavioural theoretical framework. The current study sought to determine the association between negative cognitions, within the context of Rational Emotive Behaviour Therapy (REBT), and the experience of loneliness. A multinational sample of university students ( $n=397$ ) completed self-report assessments of rational and irrational beliefs, and loneliness. Structural equation modelling results found that the REBT model of psychopathology, and the REBT model of psychological health, provided satisfactory representations of loneliness, explaining $36 \%$ and $23 \%$ of variance in loneliness, respectively. Several dysfunctional ("Demandingness", "Catastrophising" and "Self-Downing" beliefs) and functional ("Preferences" and "Self-Acceptance" beliefs) cognitions were directly and indirectly associated with loneliness. These results highlight that cognitions and loneliness are meaningfully related, and indicate that cognitive-behavioural models may be useful in understanding loneliness. More specifically, current results suggest that REBT may offer a viable psychotherapeutic approach to treating loneliness.
\end{abstract}

\section{ARTICLE HISTORY}

Received 21 February 2018

Accepted 8 May 2018

\section{KEYWORDS}

Loneliness; cognitivebehaviour therapy (CBT); Rational Emotive Behaviour Therapy (REBT); structural equation modelling (SEM)

Loneliness is an unpleasant emotional experience that can occur when an individual evaluates their life as lacking sufficient quantity or quality of social relationships (Heinrich \& Gullone, 2006). Put another way, it can be defined as an undesired absence of reciprocal empathic understanding (van Staden \& Coetzee, 2010). Large-scale general population studies across multiple nations indicate that up to $20 \%$ of people experience feelings of loneliness at any point in time (Beutel et al., 2017; Lasgaard, Friis, \& Shevlin, 2016; Stickley et al., 2013) and is commonest in early and late adulthood, and within females (Lasgaard et al., 2016). Increased loneliness has been shown to be associated with a greater likelihood 
of experiencing common mental health disorders including mood, anxiety, psychotic and personality disorders, as well as suicidal behaviour (Hawkley \& Cacioppo, 2010). Furthermore, associations extend to neurodegenerative disorders such as Alzheimer's disease and early mortality (Holt-Lunstad, Smith, \& Layton, 2010; Jaremka et al., 2013; Wilson et al., 2007).

Longitudinal studies have established that loneliness is not merely a correlate of poor psychological and physical health, but rather that loneliness precedes and predicts the onset of psychological disorders (Cacioppo, Hawkley, \& Thisted, 2010; Shevlin, McElroy, \& Murphy, 2015), suicidal ideation and behaviour (Rudatsikira, Muula, Siziya, \& TwaTwa, 2007) and physical disorders (Caspi, Harrington, Moffitt, Milne, \& Poulton, 2006; Hawkley \& Cacioppo, 2007; Shiovitz-Ezra \& Ayalon, 2010) (but see Donovan et al., 2016). Observational studies have also established that loneliness temporally precedes depressive symptomatology (Cacioppo, Hughes, Waite, Hawkley, \& Thisted, 2006) which is consistent with the theory that loneliness has a causal role to play in determining mental health outcomes. Biological mechanisms suggested to account for this causal role include dorsal raphe nuclei dysfunction (Matthews et al., 2016), inflammatory mechanisms, attractiveness, vascular health and health-related behaviours (Cacioppo et al., 2002) and increased amyloid burden (Donovan et al., 2016).

Many researchers have observed that objective social isolation alone fails to fully explain the experience of loneliness (Cacioppo \& Patrick, 2008; Peplau \& Perlman, 1982; Wheeler, Reis, \& Nezlek, 1983). Researchers have instead emphasised the role of cognitive variables such as intimacy, connectedness, negativity, threat perception and other essentially qualitative psychological elements as important influences upon loneliness, which in many cases is operationalised as perceived social isolation (Cacioppo \& Hawkley, 2009; Cacioppo et al., 2010; Hamamci \& Duy, 2007; Hawkley, Preacher, \& Cacioppo, 2011; Shevlin, Murphy, \& Murphy, 2015). Young (1982) originally discussed the clinical value of addressing negative automatic thoughts by means of cognitive behaviour therapy in order to reduce feelings of loneliness among patients suffering from depression. Since then, dysfunctional cognitions have been shown to be positively correlated with feelings of loneliness (Hyland et al., 2015), and meta-analytic findings have concluded that addressing maladaptive cognitions may be the most effective way to treat loneliness, something which has been notably intractable in the context of intervention (Masi, Chen, Hawkley, \& Cacioppo, 2011). More recently, the technique of cognitive restructuring (used frequently within a cognitive-behavioural context) was successfully used to reduce loneliness (Theeke et al., 2016). These findings suggest that traditional cognitive-behavioural models of psychopathology may offer a useful method to understand the development and maintenance of loneliness, and provide a viable psychotherapeutic method by which to reduce feelings of loneliness.

Rational Emotive Behaviour Therapy (REBT) is one such cognitive-behavioural model that may be applicable in the context of loneliness (David \& Szentagotai, 2006). REBT theory posits that specific types of negative cognitions (termed "rational" and "irrational" beliefs) relating to internal or external life events are central to the development of emotional (and behavioural and psychophysiological) responses. It is important to note that the types of negative cognitions that REBT theory posits as being central to the development of psychopathology are distinct from the negative cognitions which other cognitive-behavioural models, such as Cognitive Therapy, highlight as central to the development of psychopathology. For example, in Cognitive Therapy, negative representations of life events are 
Model A: REBT model of psychopathology.

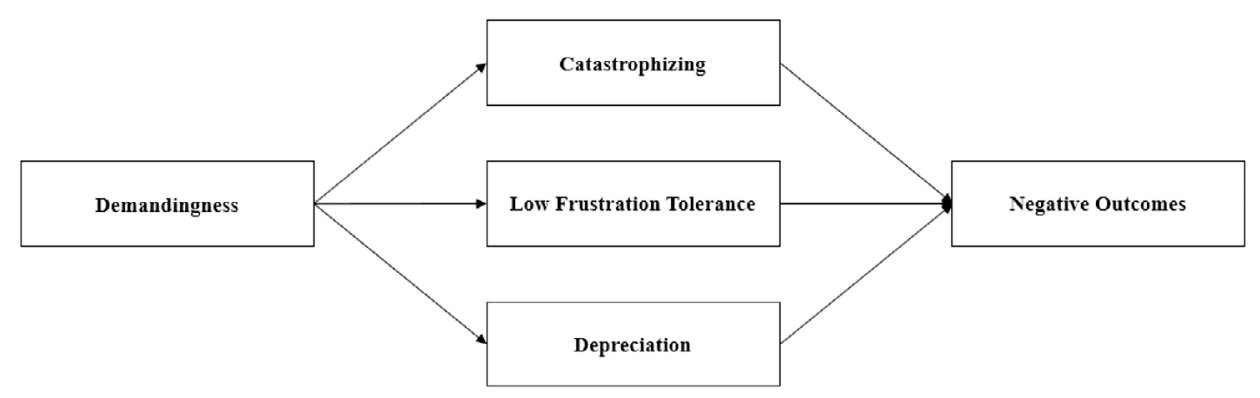

Model B: REBT model of psychological health.

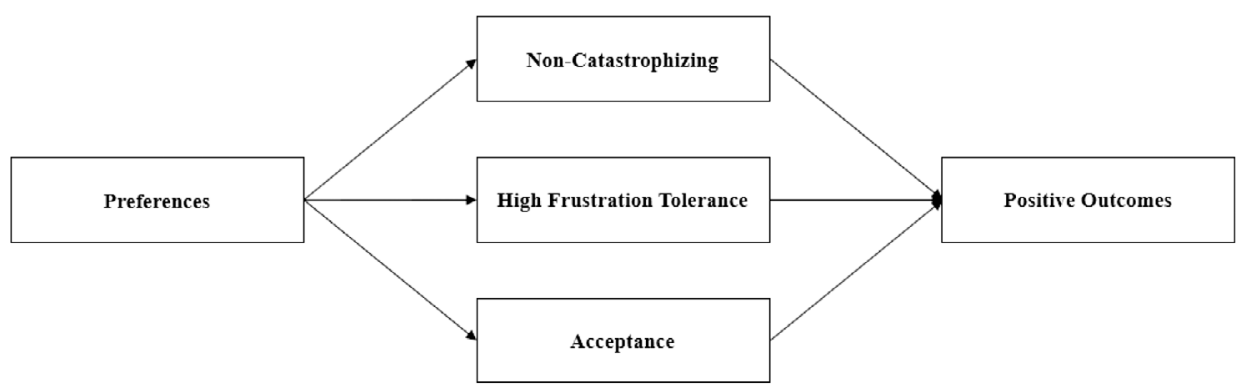

Figure 1. General representation of the REBT theory.

addressed (e.g. "negative automatic thoughts") (e.g. Young, 1982), whereas in REBT, negative evaluations of one's representations of the world are addressed (see David \& Szentagotai, 2006 for a full discussion of the qualitative differences between Cognitive Therapy and REBT). As presented in Figure 1, REBT proposes a model of psychopathology and a model of psychological health.

The REBT model of psychopathology focuses on four "irrational" beliefs: (1) "Demandingness" beliefs (rigid insistences for how oneself, others, or the world must be); (2) "Catastrophising" beliefs (extreme and unrealistic negative evaluations of an event or situation); (3) "Low Frustration Tolerance" beliefs (extreme and unrealistic negative evaluations of one's own capacity to tolerate or withstand an event of situation); and (4) "Depreciation" beliefs (extreme and unrealistically negative evaluations of oneself, others, or the world). This model has been found to explain the occurrence of a range of mental health problems, including depression, anxiety and posttraumatic stress disorder (David, Schnur, \& Belloiu, 2002; Hyland, Shevlin, Adamson, \& Boduszek, 2014a; Nieuwenhuijsen, Verbeek, Boer, Blonk, \& van Dijk, 2010).

The REBT model of psychological health focuses on four "rational" beliefs: (1) "Preference" beliefs (flexible desires directed towards the self, others and the world); (2) "Non-Catastrophising" beliefs (realistic evaluations of the negativity of an event or situation); (3) "High Frustration Tolerance" beliefs (realistic evaluations of one's own capacity to tolerate or withstand an event or situation); and (4) "Acceptance beliefs" (realistic and accepting evaluations of oneself, others and the world). Although not as frequently 
investigated as the REBT model of psychopathology, the REBT model of psychological health has been shown to protect against the development of posttraumatic stress responses (Hyland, Maguire, Shevlin, \& Boduszek, 2014), and depression and anxiety responses (Oltean, Hyland, Vallières, \& David, 2017).

Although Young (1982) proposed the value of recognising loneliness as a viable clinical target for CBT over 35 years ago, a coherent CBT-based theoretical model of loneliness has not yet been conceptualised. It is our belief however that loneliness can be effectively understood within the theoretical framework of REBT. Moreover, by potentially identifying specific cognitive vulnerability factors for loneliness (i.e. the REBT model of psychopathology), as well as cognitive protective factors against loneliness (i.e. the REBT model of psychological health), an important advance can be made in (1) providing a theoretical account of loneliness, and (2) developing theoretically derived psychotherapeutic interventions for loneliness that are based upon an empirically supported model of psychotherapy (David, Cotet, Matu, Mogoase, \& Stefan, 2018). The primary objective of the current study therefore was to assess the validity of REBT's psychopathology and psychological health models to explain loneliness. Structural equation modelling (SEM) techniques were used to assessed the validity of these models among a multinational sample of university students; a cohort that is recognised to represent high-risk for feelings of loneliness (Lasgaard et al., 2016). It was hypothesised that both the REBT model of psychopathology and the REBT model of psychological health would effectively explain observed variation in feelings of loneliness.

\section{Methods}

\section{Participants and procedures}

The sample for the current study was comprised of undergraduate psychology and computer science students recruited from four universities in the Republic of Ireland, Northern Ireland and England $(N=397)$. The sample included a similar number of males $(n=191$, $49.6 \%)$ and females $(n=194,50.4 \%)$ with an average age of 23.33 years (SD $=7.91$, range $18-60)$. Students had spent 2.23 years on average in third-level education $(S D=1.20$, range $0-7$ years) at the time of assessment. The majority of students lived in urban or suburban environments $(n=252,65.7 \%)$ and were single ( $n=315,82 \%)$.

All participants were sampled opportunistically and data was collected during the academic calendar from September 2013 to May 2014. Ethical approval was obtained from the ethical review board at the National College of Ireland. All students under the age of 18 were excluded from the study. Participants were assured of confidentiality, instructed that they were under no obligation to participate and could withdraw at any time. Participants completed the questionnaires using a paper-and-pencil format in their regular lecture theatre setting. No inducements or incentives were used to recruit volunteers.

\section{Measures}

The Attitudes and Belief Scale 2-Abbreviated Version (ABS2-AV: Hyland, Shevlin, Adamson, \& Boduszek, 2014b) is a 24-item self-report measure of rational (Preferences, Non-Catastrophising, High Frustration Tolerance and Self-Acceptance) and irrational (Demandingness, Catastrophising, Low Frustration Tolerance and Self-Downing) beliefs. 
Each rational and irrational belief process is measured via three items and all items are scored using a five-point Likert-scale from 1 (Strongly Disagree) to 5 (Strongly Agree). Higher scores in each case indicate higher levels of each belief process. Previous psychometric assessments of the ABS2-AV found that the questionnaire possesses satisfactory factorial validity and internal reliability (Hyland, Shevlin, et al., 2014b). Among the current sample, internal reliability estimates (Cronbach's alpha) for three of the four irrational belief processes were slightly lower than desirable (Demandingness $=.68$; Catastrophising = .67; Low Frustration Tolerance $=.65$; and Self-Downing $=.75)$. The reliability estimates for the four rational belief processes were mixed; Preferences (.74) and Self-Acceptance (.77) beliefs demonstrated satisfactory reliability, whereas Non-Catastrophising (.36) and High Frustration Tolerance (.52) beliefs possessed poor reliability.

Feelings of loneliness were measured using the Three-Item Loneliness Scale (ULS-3; Hughes, Waite, Hawley, \& Cacioppo, 2004). The ULS-3 is a shortened form of the widely used 20-item Revised-UCLA Loneliness Scale (R-UCLA: Russell, Peplau, \& Cutrona, 1980). The ULS-3 was developed in order to be used as an effective screening measure of loneliness in large-scale research studies. The three items that comprise the ULS-3 were selected from the R-UCLA based on an exploratory factor analysis of a large, nationally representative sample of the United States population. The three items are: (i) "I feel left out", (ii) "I feel isolated", and (iii) "I lack companionship". These items were measured using a four-point Likert scale ( 1 = I never feel this way, 2 = I rarely feel this way, 3 = I sometimes feel this way, $4=$ I often feel this way), and higher scores reflect higher levels of loneliness. The psychometric properties of the ULS-3 were established among two distinct nationally representative samples of the United States population, with both studies yielding good internal reliability results (Cronbach's alpha's in both studies were .72), strong associations with scores on the R-UCLA ( $\mathrm{r}=.82$ ), and good convergent and divergent validity (see Hughes et al., 2004). Cronbach's alpha for the ULS-3 among the current sample was .83.

\section{Data analysis}

Structural equation modelling (SEM) was used to test the hypothesised REBT psychopathology and psychological health models of loneliness (see Figures 2 and 3). SEM is a combination of two analytic procedures: confirmatory factor analysis (CFA), which assesses the measurement component of a theoretical model, and path analysis, which assesses the relationships between latent variables within a model. A number of features make the use of SEM appropriate for the current analysis: (1) the ability to control for measurement error which improves the reliability of results, (2) the ability to test the congruence between the hypothesised model structures and the sample data which allows for falsification of the proposed model structures, and (3) the ability to simultaneous test for direct, indirect and total effects between the variables in the model (Bollen, 1989; Kline, 2011). The recommendations of Anderson and Gerbing (1988) were followed and a two-stage modelling procedure was conducted. The validity of the measurement models were first established, followed by an assessment of the structural models.

In order to test the validity of the measurement and structural components of each model, standard recommendations for determination of model fit were followed: non-significant chi-square; CFI and TLI values > .90, and RMSEA and SRMR values $<.08$ ( Hu \& Bentler, 1999). In order to test for indirect effects in both models, the recommendations of Preacher 


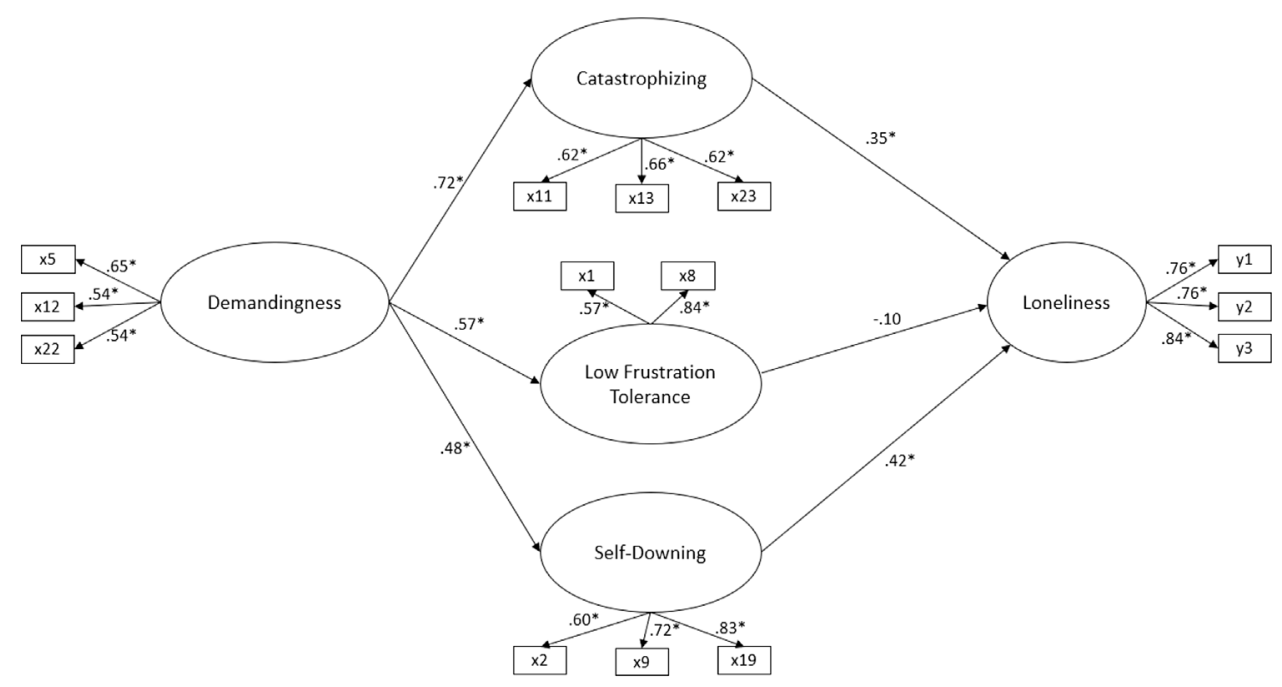

Figure 2. REBT Psychopathology model of loneliness.

Notes: Statistical significance, ${ }^{*}$ indicates $p<.001 ; x 1-x 23$ refers to the irrational belief items of the ABS2-AV; $y 1-y 3$ refers to the three items of the ULS-3.

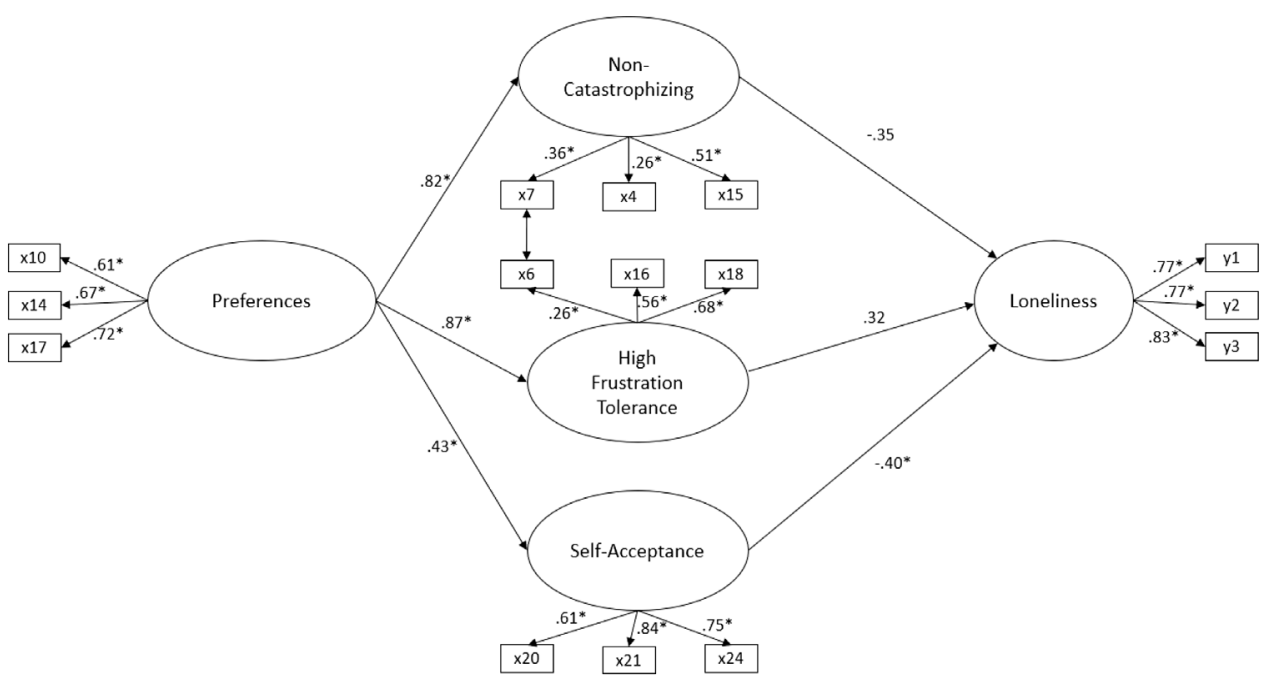

Figure 3. REBT Psychological health model of loneliness.

Notes: Statistical significance, ${ }^{*}$ indicates $p<.001 ; \mathrm{x} 4-\mathrm{x} 24$ refers to the irrational belief items of the ABS2-AV; $\mathrm{y} 1-\mathrm{y} 3$ refers to the three items of the ULS-3.

and Hayes (2008) were followed and bias-corrected (BC) bootstrapping techniques were applied. Bootstrapping is a non-parametric resampling technique that does not assume multivariate normality of the sampling distribution, and allows for the production of confidence intervals around the observed indirect effects. To produce confidence intervals for the indirect effects 1,000 bootstrap samples were used. The CFA procedures were estimated using Robust Maximum Likelihood estimation (MLR: Yuan \& Bentler, 2000), however for the SEM analyses Maximum Likelihood estimation was used as the MLR estimator cannot 
Table 1. Descriptive statistics for all continuous variables.

\begin{tabular}{lcrrcc}
\hline & Mean (95\% Confidence Intervals) & Median & SD & Range & Possible Range \\
\hline Loneliness & $5.36(5.13-5.60)$ & 5.00 & 2.29 & $3-12$ & $3-12$ \\
Demandingness & $10.52(10.27-10.77)$ & 11.00 & 2.45 & $3-15$ & $3-15$ \\
Catastrophising & $8.17(7.88-8.46)$ & 8.00 & 2.88 & $3-15$ & $3-15$ \\
Low frustration tolerance & $9.49(9.22-9.75)$ & 10.00 & 2.59 & $3-15$ & $3-15$ \\
Self-downing & $5.15(4.90-5.40)$ & 4.00 & 2.47 & $3-15$ & $3-15$ \\
Preferences & $9.35(9.09-9.62)$ & 9.00 & 2.60 & $3-15$ & $3-15$ \\
Non-catastrophising & $11.06(10.87-11.26)$ & 11.00 & 1.93 & $5-15$ & $3-15$ \\
High frustration tolerance & $10.31(10.08-10.53)$ & 11.00 & 2.25 & $3-15$ & $3-15$ \\
Self-acceptance & $11.95(11.69-12.22)$ & 12.00 & 6.98 & $3-15$ & $3-15$ \\
\hline
\end{tabular}

be used when applying bootstrapping techniques. Missing data was low (1.3\%) and analyses were based on all available information. Analyses were conducted in Mplus version 7.4 (Muthén \& Muthén, 1998-2013).

\section{Results}

\section{Descriptive statistics}

Measures of central tendency and variability for loneliness and each of the irrational and rational belief processes are displayed in Table 1.

\section{Step 1: measurement models}

\section{Measurement model of loneliness}

In order to conduct a CFA on the ULS-3 it was necessary to constrain the residual variance of items 1 and 2 to be equal in order to avoid a just-identified model. The unidimensional structure of the ULS-3 was supported $\left(\chi^{2}=.56, \mathrm{df}=1, p=.45 ; \mathrm{CFI}=1.00\right.$; TLI = 1.01; RMSEA $=.00(95 \% \mathrm{CI}=.00-.12)$; SRMR $=.01)$. This latent variable of loneliness was included as the endogenous variable in both the REBT psychopathology and psychological health models.

\section{Measurement model of irrational and rational beliefs}

The factorial structure of the ABS2-AV was comprehensively evaluated amongst the current sample in a prior study (Hyland et al., 2017). Using CFA procedures within an alternative models framework design, the best fitting model included eight latent factors: four factors measuring each irrational belief process (Demandingness, Catastrophising, Low Frustration Tolerance and Self-Downing), and four factors measuring each rational belief process (Preferences, Non-Catastrophising, High Frustration Tolerance and Self-Acceptance). Two modifications to the factorial model were required to achieve satisfactory fit. First, one item which was intended to measure Low Frustration Tolerance beliefs ("It's unbearable to fail at important things, and I can't stand not succeeding at them") was found to possess a very high cross-factor loading on the Demandingness factor therefore this item was removed. Second, a large residual covariance existed between one Non-Catastrophising item ("When life is hard and I feel uncomfortable, I realise it is not awful to feel uncomfortable or tense, only unfortunate and I can keep going") and one High Frustration Tolerance item ("I do not like to be uncomfortable, tense or nervous, but I can tolerate being tense"). The high 
residual covariance between these two items was understandable given the fact that both items present the respective rational belief processes in the context of being uncomfortable, therefore, a residual covariance between these two items were included. With these two modifications to the measurement of rational and irrational beliefs the fit of the model was satisfactory $\left(\chi^{2}=397.01, \mathrm{df}=222, p<.001\right.$; CFI $=.92$; TLI $=.90$; RMSEA $=.05(95 \%$ $\mathrm{CI}=.04-.05)$; SRMR $=.05)$. All model parameters were positive, statistically significant $(p<.001)$, and of a robust magnitude. Latent variables representing the four irrational and four rational beliefs were included as exogenous variables within the REBT psychopathology and psychological health models of loneliness, respectively (see Figures 2 and 3).

\section{Step 2: structural models}

\section{REBT psychopathology model of loneliness}

The REBT psychopathology model of loneliness (Figure 2) provided an acceptable fit to the data $\left(\chi^{2}=186.01, \mathrm{df}=71, p<.001 ; \mathrm{CFI}=.93\right.$; TLI $=.91$; $\mathrm{RMSEA}=.06(95 \% \mathrm{CI}=.05-.08)$; SRMR $=.06)$ and explained $36 \%$ of variance in loneliness. Parameter estimates indicated that Demandingness beliefs were strongly associated with Catastrophising $(\beta=.72, p=.001)$, Low Frustration Tolerance $(\beta=.57, p=.013)$ and Self-Downing $(\beta=.48, p=.008)$ beliefs. Catastrophising beliefs $(\beta=.35, p=.001)$ and Self-Downing beliefs $(\beta=.42, p<.001)$ were positively associated with loneliness.

The indirect relationship between Demandingness beliefs and loneliness via (i) Catastrophising beliefs $(\beta=.25, \mathrm{SE}=.08,95 \% \mathrm{CI}(\mathrm{BC})=.09$ to $.41, p=.003)$ and (ii) Self-Downing beliefs $(\beta=.20, \mathrm{SE}=.05,95 \% \mathrm{CI}(\mathrm{BC})=.10$ to $.30, p<.001)$ were statistically significant and of a robust magnitude. It can be concluded therefore that increased levels of Demandingness beliefs are indirectly related to increased levels of loneliness via Catastrophising beliefs and Self-Downing beliefs.

\section{REBT psychological health model of loneliness}

The REBT psychological health model of loneliness (Figure 3) provided an acceptable representation of the sample data $\left(\chi^{2}=185.60, \mathrm{df}=83, p<.001\right.$; CFI $=.93$; TLI $=.92$; RMSEA $=.06(95 \% \mathrm{CI}=.05-.07)$; SRMR $=.06)$, and explained $23 \%$ of variance in loneliness. Parameter estimates indicated that Preference beliefs were significantly associated with Non-Catastrophising $(\beta=.82, p=.001)$, High Frustration Tolerance $(\beta=.87, p=.006)$, and Self-Acceptance $(\beta=.43, p<.001)$ beliefs. Self-Acceptance beliefs were negatively associated with loneliness $(\beta=-.40, p<.001)$.

The indirect relationship between Preference beliefs and loneliness via Self-Acceptance beliefs was statistically significant $(\beta=-.17, \mathrm{SE}=.05,95 \% \mathrm{CI}(\mathrm{BC})=-.26$ to $-.08, p<.001)$. This indicates that increased levels of Preference beliefs are indirectly associated with lower levels of loneliness via Self-Acceptance beliefs.

\section{Discussion}

The current study was carried out to determine the association between negative evaluative cognitions and feelings of loneliness using the REBT theoretical framework. The REBT models of psychopathology and psychological health, respectively, provided viable theoretical frameworks in which to understand the experience of loneliness among a multinational 
sample of young adults attending university. SEM results supported the study hypothesis as the REBT models of psychopathology and psychological health were found to provide satisfactory model fit, and explained 36 and $23 \%$ of variance in loneliness, respectively. Evidently, a model comprised of dysfunctional cognitions (i.e. negative evaluations or "irrational beliefs") provided a better explanation of variation in feelings of loneliness than a model comprised of functional cognitions (i.e. realistic evaluations or "rational beliefs"). Nonetheless, the REBT psychological health model did explain a meaningful level of variation in feelings of loneliness, and rational beliefs were found to be robustly associated with loneliness. The REBT psychological health model would also predict that, in addition to being negatively related to feelings of loneliness (a negative emotional consequence), rational beliefs should also be positively associated with functional social processes such as social integration and quality of social relationships (positive emotional and behavioural consequences). It is important that future research addresses this specific prediction of the model.

The SEM findings indicated that Demandingness beliefs had an indirect effect on loneliness via (i) Catastrophising beliefs and (ii) Self-Downing beliefs. Individuals high in Demandingness are also higher in loneliness, and this appears to be in part due to their higher levels of Catastrophising and Self-Downing beliefs. No such pathway was found between Demandingness and loneliness via Low Frustration Tolerance beliefs, however. Within the REBT psychological health model, Preference beliefs were related to loneliness via their effect on Self-Acceptance beliefs. Individuals high in Preference beliefs were lower in loneliness, due in part, seemingly, to their higher levels of Self-Acceptance. Current results provide novel and additional support for the general REBT theory of psychopathology and psychological health by demonstrating, for the first time, that irrational and rational beliefs, which are established correlates of common psychiatric disorders (e.g. David et al., 2002; Hyland, Maguire, et al., 2014; Hyland, Shevlin, et al., 2014a; Nieuwenhuijsen et al., 2010; Oltean et al., 2017), are also associated with feelings of loneliness.

Taken together these results have a number of important theoretical and clinical implications. Theoretically, current results add to a growing literature attesting to the important associations between dysfunctional cognitive processes and feelings of loneliness (e.g. Cacioppo et al., 2010; Hyland et al., 2015; Shevlin, McElroy, et al. 2015; Young, 1982). The current study found evidence that certain dysfunctional (Demandingness, Catastrophising and Self-Downing) and functional (Preferences and Self-Acceptance) beliefs are associated with loneliness thus contributing to current understandings of the specific cognitive correlates of loneliness. Clinically, these findings suggest that REBT might provide a useful framework to conceptualise, understand and treat feelings of loneliness. Meta-analytic findings show that REBT is an effective treatment for a range of psychologically distressing issues (David et al., 2018) however it will be important for future research to determine if REBT can also effectively reduce feelings of loneliness. While REBT may well offer a viable theoretical and clinical framework in which to understand and treat loneliness, the development of a more comprehensive and integrated cognitive-behavioural theory of loneliness - one which recognises other important cognitive variables such as negative automatic thoughts (Young, 1982) - would likely produce the most effect interventions to tackle loneliness and promote mental and physical wellbeing. (Hawkley \& Cacioppo, 2010). Given that loneliness is associated with (Hawkley \& Cacioppo, 2010), and predictive of (Cacioppo et al., 2006, 2010; Shevlin, McElroy, et al. 2015), a range of debilitating and costly mental and neurodegenerative disorders, the development of an effective psychological treatment for loneliness would 
consequently lead to substantial reductions in the global burden and financial impact associated with such disorders (Olesen et al., 2012).

There are several limitations associated with this study which should be recognised. First, two of the rational belief subscales (Non-Catastrophising beliefs and High Frustration Tolerance beliefs) exhibited less than satisfactory reliability, and although the use of SEM allowed errors in measurement to be accounted for, the issue of appropriate measurement of rational and irrational beliefs remains an important issue in REBT research (see Hyland et al., 2017). Relatedly, with respect to "Depreciation" and "Acceptance" beliefs, the ABS2-AV only captures these constructs as they relate to the self, and not as they relate to others or the world in general. How such cognitive evaluations might relate to feelings of loneliness remains an important unaddressed question. Second, results are based on a convenience sample of undergraduate students and overall levels of loneliness were low. The generalisability of current findings to other populations, particularly clinical populations, is questionable. Nevertheless, current results are supportive of the role of cognitive-behavioural models in understanding the subjective experience of loneliness within a cohort of the population that are particularly at-risk of feelings of loneliness (Lasgaard et al., 2016). Finally, since data were cross-sectional in nature no assessments of mediation, which the REBT theory implies, could be tested. Moreover, it is possible that loneliness may be the causal factor in the model, rather than the cognitions. This possibility makes sense if loneliness is framed as a personality trait, relatively stable across the lifespan, as has been suggested (Shiovitz-Ezra \& Ayalon, 2010). Importantly, REBT theory is flexible enough to incorporate this possibility as the theory posits that emotional consequences (i.e. feelings of loneliness) can become new activating events which could be subsequently evaluated by means of rational or irrational beliefs. Longitudinal research is required to effectively test the temporal associations between cognitions and loneliness, and cross-lagged panel modelling would be an ideal statistical design to determine if loneliness and cognitions reciprocally interact across time.

In conclusion, results provide initial evidence that loneliness, a common psychological experience that is robustly associated with several psychological and neurodegenerative disorders, may be effectively conceptualised within a cognitive-behavioural theoretical framework; specifically the REBT theoretical framework. Results are compatible with previous assertions that tackling dysfunctional cognitions may be the best way to reduce loneliness (Masi et al., 2011; Theeke et al., 2016). Recommendations could be made arising from the current results to develop interventions for loneliness along REBT principles. Currently, there is a dearth of evidence concerning effective ways in which to treat loneliness in at-risk populations. The current study represents a possible way to respond to recommendations made by Masi et al. (2011) and develop cognitive interventions to tackle the problem of loneliness.

\section{Acknowledgements}

Following the sad news of the recent death of John Cacioppo, we would like to acknowledge the incredibly valuable contribution that his life's work had on the field of loneliness research. John's work was pioneering and it raised the consciousness of innumerable researchers and clinicians to the importance of loneliness. In doing so, John's work inspired a revolution across many fields of scientific inquiry. As one very minor example, this study would not have been possible had it not been for his incredible work. John Cacioppo's death is an enormous loss to the field and his absence will be felt. 


\section{Disclosure statement}

No potential conflict of interest was reported by the authors.

\section{ORCID}

Philip Hyland (D) http://orcid.org/0000-0002-9574-7128

Jamie Murphy (iD) http://orcid.org/0000-0003-1821-0025

Frédérique Vallières (iD http://orcid.org/0000-0001-6315-3029

\section{References}

Anderson, J. C., \& Gerbing, D. W. (1988). Structural equation modeling in practice: A review and recommended two-step approach. Psychological Bulletin, 103, 411-423. https://doi. org/10.1037/0033-2909.103.3.411

Bollen, K. A. (1989). Structural equations with latent variables. New York, NY: Wiley. https://doi. org/10.1002/9781118619179

Beutel, M. E., Klein, E. M., Brähler, E., Reiner, I., Jünger, C., Michal, M., ... Tibubos, A. N. (2017). Loneliness in the general population: Prevalence, determinants and relations to mental health. BMC Psychiatry, 17, 97. https://doi.org/10.1186/s12888-017-1262-x

Cacioppo, J. T., \& Hawkley, L. C. (2009). Perceived social isolation and cognition. Trends in Cognitive Sciences, 13, 447-454. https://doi.org/10.1016/j.tics.2009.06.005

Cacioppo, J. T., Hawkley, L. C., Crawford, L. E., Ernst, J. M., Burleson, M. H., \& Kowalewski, R. B. (2002). Loneliness and health: Potential mechanisms. Psychosomatic Medicine, 64, 407-417.

Cacioppo, J. T., Hawkley, L., \& Thisted, R. A. (2010). Perceived social isolation makes me sad: 5-Year cross-lagged analyses of loneliness and depressive symptomatology in the Chicago health, aging, and social relations study. Psychology and Aging, 25(2), 453-463. https://doi.org/10.1037/a0017216

Cacioppo, J. T., Hughes, M. E., Waite, L. J., Hawkley, L. C., \& Thisted, R. A. (2006). Loneliness as a specific risk factor for depressive symptoms: Cross-sectional and longitudinal analyses. Psychology and Aging, 21(1), 140-151. https://doi.org/10.1037/0882-7974.21.1.140

Cacioppo, J. T., \& Patrick, W. (2008). Loneliness: Human nature and the need for social connection. New York, NY: Norton.

Caspi, A., Harrington, H., Moffitt, T. E., Milne, B. J., \& Poulton, R. (2006). Socially isolated children 20 years later: risk of cardiovascular disease. Archives of Pediatrics \& Adolescent Medicine, 160, 805-811.

David, D., Cotet, C., Matu, S., Mogoase, C., \& Stefan, S. (2018). 50 years of rational-emotive and cognitive-behavioral therapy: A systematic review and meta-analysis. Journal of Clinical Psychology, 74, 304-318. https://doi.org/10.1002/jclp.22514

David, D., Schnur, J., \& Belloiu, A. (2002). Another search for the "hot" cognitions: Appraisal, irrational beliefs, attributions, and their relation to emotion. Journal of Rational-Emotive and Cognitive-Behavior Therapy, 15, 93-131.

David, D., \& Szentagotai, A. (2006). Cognition in cognitive-behavioral psychotherapies (CBT): Toward an integrative model. Clinical Psychology Review, 3, 284-298.

Donovan, N. J., Okereke, O. I., Vannini, P., Amariglio, R. E., Rentz, D. M., Marshall, G. A., ... Sperling, R. A. (2016). Association of higher cortical amyloid burden with loneliness in cognitively normal older adults. JAMA Psychiatry, 73(12), 1230-1237. https://doi.org/10.1001/jamapsychiatry.2016.2657

Hamamci, Z., \& Duy, B. (2007). The relationship among social skills, dysfunctional attitudes, irrational beliefs, interpersonal cognitive distortions and loneliness. Eurasian Journal of Educational Research, 26, 121-130.

Hawkley, L. C., \& Cacioppo, J. T. (2007). Aging and loneliness: Downhill quickly? Current Directions in Psychological Science, 16, 187-191. 
Hawkley, L. C., \& Cacioppo, J. T. (2010). Loneliness matters: A theoretical and empirical review of consequences and mechanisms. Annals of Behavioral Medicine, 40, 218-27. https://doi.org/10.1007/ s12160-010-9210-8

Hawkley, L., Preacher, K., \& Cacioppo, J. (2011). As we said, loneliness (not living alone) explains individual differences in sleep quality: Reply. Health Psychology, 30, 136. https://doi.org/10.1037/ a0022366

Heinrich, L. M., \& Gullone, E. (2006). The clinical significance of loneliness: A literature review. Clinical Psychology Review, 26, 695-718. https://doi.org/10.1016/j.cpr.2006.04.002

Holt-Lunstad, J., Smith, T. B., \& Layton, J. B. (2010). Social relationships and mortality risk: A metaanalytic review. PLoS Med, 7, e1000316. https://doi.org/10.1371/journal.pmed.1000316

Hughes, M. E., Waite, L. J., Hawley, L. C., \& Cacioppo, J. T. (2004). A short scale for measuring loneliness in large surveys. Research on Aging, 26, 655-672. https://doi.org/10.1177/0164027504268574

Hu, L., \& Bentler, P. (1999). Cutoff criteria for fit indices in covariance structure analysis: Conventional criteria versus new alternatives. Structural Equation Modeling, 6, 1-55. https://doi. org/10.1080/10705519909540118

Hyland, P., Maguire, R., Shevlin, M., \& Boduszek, D. (2014). Rational beliefs as cognitive protective factors against posttraumatic stress symptoms. Journal of Rational-Emotive \& Cognitive-Behaviour Therapy, 32, 297-312. https://doi.org/10.1007/s10942-014-0195-2

Hyland, P., Shevlin, M., Adamson, G., \& Boduszek, B. (2014a). The organization of irrational beliefs in posttraumatic stress symptomology: Testing the predictions of REBT theory using structural equation modelling. Journal of Clinical Psychology, 70, 48-59. https://doi.org/10.1002/jclp.22009

Hyland, P., Shevlin, M., Adamson, G., \& Boduszek, D. (2014). Modelling the structure of the attitudes and belief scale 2: Toward the development of an abbreviated version. Cognitive Behaviour Therapy, 43, 60-71. https://doi.org/10.1080/16506073.2013.777467

Hyland, P., Murphy, J., Shevlin, M., Murphy, S., Egan, A., \& Boduszek, D. (2015). Psychometric properties of the posttraumatic cognition inventory within a Northern Ireland adolescent sample. British Journal of Clinical Psychology, 54, 435-449. https://doi.org/10.1111/bjc.12089

Hyland, P., Fox, R., Treacy, G., Maguire, P., Boduszek, D., \& Vallières, F. (2017). Assessing the factorial validity of the attitudes and belief scale 2 -abbreviated version: A call for the development a gold standard method of measuring rational and irrational beliefs. Journal of Rational-Emotive \& Cognitive-Behaviour Therapy, 35, 111-124. https://doi.org/10.1007/s10942-016-0243-1

Jaremka, L. M., Andridge, R. R., Alfano, C. M., Povoski, S. P., Lipari, A. M., Agnesse, D. M., ... KieoltGlaser, J. K. (2013). Pain, depression, and fatigue: Loneliness as a longitudinal risk factor. Health Psychology, 33(9), 948-957. https://doi.org/10.1037/a0034012

Kline, R. B. (2011). Principles and practice of structural equation modeling (3rd ed.). London: The Guilford Press.

Lasgaard, M., Friis, K., \& Shevlin, M. (2016). “Where are all the lonely people?” A population-based study of high-risk groups across the life span. Social Psychiatry and Psychiatric Epidemiology, 51, 1373-1384. https://doi.org/10.1007/s00127-016-1279-3

Masi, C., Chen, H., Hawkley, L. C., \& Cacioppo, J. (2011). A meta-analysis of interventions to reduce loneliness. Personality and Social Psychology Review, 15, 219-266. https://doi. org/10.1177/1088868310377394

Matthews, G. A., Nieh, E. H., Vander Weele, C. M., Halbert, S. A., Pradhan, R. V., Yosafat, A. S., ... Wildes, C. P. (2016). Dorsal raphe dopamine neurons represent the experience of social isolation. Cell, 164, 617-631. https://doi.org/10.1016/j.cell.2015.12.040

Muthén, L. K., \& Muthén, B. O. (1998-2013). MPlus user's guide (7th ed.). Los Angeles, CA: Muthén \& Muthén.

Nieuwenhuijsen, K., Verbeek, J. H. A. M., Boer, A. G. E. M., Blonk, R. W. B., \& van Dijk, F. J. H. (2010). Irrational beliefs in employees with an adjustment, a depressive, or an anxiety disorder: A prospective cohort study. Journal of Rational-Emotive \& Cognitive-Behavior Therapy, 28, 57-72.

Olesen, J., Gustavsson, A., Svensson, M., Wittchen, H. U., Jönsson, B., CDBE2010 study group, \& European Brain Council. (2012). The economic cost of brain disorders in Europe. European Journal of Neurology, 19, 155-162. https://doi.org/10.1111/j.1468-1331.2011.03590.x 
Oltean, H. R., Hyland, P., Vallières, F., \& David, D. O. (2017). An empirical assessment of REBT models of psychopathology and psychological health in the prediction of anxiety and depression symptoms. Behavioural and Cognitive Psychotherapy, 45, 600-615. https://doi.org/10.1017/ S1352465817000133.

Peplau, L. A., \& Perlman, D. (1982). Loneliness: A sourcebook of current theory, research, and therapy. New York, NY: Wiley.

Preacher, K. J., \& Hayes, A. F. (2008). Asymptotic and resampling strategies for assessing and comparing indirect effects in multiple mediator models. Behavior Research Methods, 40, 879-891. https://doi.org/10.3758/BRM.40.3.879

Rudatsikira, E., Muula, A. S., Siziya, S., \& Twa-Twa, J. (2007). Suicidal ideation and associated factors among school-going adolescents in rural Uganda. BMC Psychiatry, 67, 1-6.

Russell, D., Peplau, L. A., \& Cutrona, C. E. (1980). The revised UCLA loneliness scale: Concurrent and discriminant validity evidence. Journal of Personality and Social Psychology, 39, 472-480. https://doi.org/10.1037/0022-3514.39.3.472

Shevlin, M., McElroy, E., \& Murphy, J. (2015). Loneliness mediates the relationship between childhood trauma and adult psychopathology: Evidence from the adult psychiatric morbidity survey. Social Psychiatry and Psychiatric Epidemiology, 50, 591-601. https://doi.org/10.1007/s00127-014-0951-8

Shevlin, M., Murphy, S., \& Murphy, J. (2015). The latent structure of loneliness: Testing competing factor models of the UCLA Loneliness Scale in a large adolescent sample. Assessment, 22, 208-15. https://doi.org/10.1177/1073191114542596

Shiovitz-Ezra, S., \& Ayalon, L. (2010). Situational versus chronic loneliness as risk factors for all-cause mortality. International Psychogeriatrics, 22, 455-462. https://doi.org/10.1017/S1041610209991426

van Staden, C. W., \& Coetzee, K. (2010). Conceptual relations between loneliness and culture. Current Opinion in Psychiatry, 23(1), 524-529. https://doi.org/10.1097/YCO.0b013e32833f2ff9

Stickley, A., Koyanagi, A., Roberts, B., Richardson, E., Abbott, P., Tumanov, S., \& McKee, M. (2013). Loneliness: Its correlates and association with health behaviours and outcomes in nine countries of the former Soviet Union. PLoS ONE, 8, e67978. https://doi.org/10.1371/journal.pone.0067978

Theeke, L. A., Mallow, J. A., Moore, J., McBurney, A., Rellick, S., \& van Gilder, R. (2016). Effectiveness of LISTEN on loneliness, neuroimmunological stress response, psychosocial functioning, quality of life, and physical health measures of chronic illness. International Journal of Nursing Sciences, 3, 242-251. https://doi.org/10.1016/j.ijnss.2016.08.004

Wheeler, L., Reis, H., \& Nezlek, J. (1983). Loneliness, social interaction, and sex roles. Journal of Personality and Social Psychology, 45, 943-953. https://doi.org/10.1037/0022-3514.45.4.943

Wilson, R. S., Krueger, K. R., Arnold, S. E., Schneider, J. A., Kelly, J. F., Barnes, L. L., ... Bennett, D. A. (2007). Loneliness and risk of alzheimer disease. Archives of General Psychiatry, 64, 234-240. https://doi.org/10.1001/archpsyc.64.2.234

Young, J. E. (1982). Loneliness, Depression and Cognitive Therapy: Theory and Application. In L. A. Peplau \& D. Perlman (Eds.), Loneliness: A Sourcebook of Current Theory, Research and Therapy (pp. 1-18). New York, NY: Wiley.

Yuan, K. H., \& Bentler, P. M. (2000). Three likelihood-based methods for mean and covariance structure analysis with nonnormal missing data. Sociological Methodology, 30, 165-200. https:// doi.org/10.1111/0081-1750.00078 